\title{
MicroRNA miR-451 downregulates the PI3K/AKT pathway through CAB39 in human glioma
}

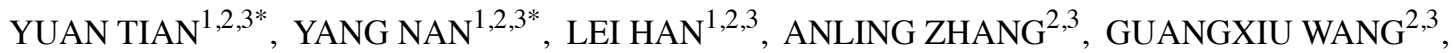 \\ ZHIFAN JIA ${ }^{2,3}$, JIANWEI HAO ${ }^{1,2,3}$, PEIYU PU ${ }^{2,3}$, YUE ZHONG ${ }^{1}$ and CHUNSHENG KANG ${ }^{1,2,3}$ \\ ${ }^{1}$ Department of Neurosurgery, Tianjin Medical University General Hospital; ${ }^{2}$ Laboratory of Neuro- \\ Oncology, Tianjin Neurological Institute; ${ }^{3}$ Key Laboratory of Neurotrauma, Variation and Regeneration, \\ Ministry of Education and Tianjin Municipal Government, Tianjin 300052, P.R. China
}

Received September 28, 2011; Accepted November 30, 2011

DOI: $10.3892 /$ ijo.2011.1306

\begin{abstract}
The microRNA miR-451 is downregulated in gliomas, this has been suggested by several different research groups and is consistent with our data. Our previous study also confirmed that miR-451 has a repressive role in glioma by inhibiting cell growth, proliferation and by inducing cell apoptosis. In the present study, we identified a target gene of miR-451 in human glioma and investigated the mechanism for the glioma suppressive effect of miR-451 functions. Expression of miR-451 in gliomas was identified by quantitative real-time PCR and fluorescence in situ hybridization. Human glioma cell lines (U251, U87, LN229 and A172) were transfected with miR-451 mimics to restore miR-451 expression. The tumor suppressive effects of miR-451 were further verified by subcutaneous assays in nude mice, in addition to our previous in vitro data. A candidate target gene was tested by Western blotting and luciferase reporter assays. Some PI3K/AKT pathway factors were tested by Western blotting. We found that miR-451 expression was downregulated in glioma samples and was inversely correlated with WHO grades of gliomas. In vivo assays confirmed that miR-451 had tumor suppressive traits. CAB39-3'UTR luciferase reporter assay confirmed CAB39 as a direct target gene of miR-451. Significant alterations in the expression of PI3K/ AKT pathway factors were observed by Western blot assays. We conclude that miR-451 represses glioma in vitro and in vivo,
\end{abstract}

Correspondence to: Dr Yue Zhong, Department of Neurosurgery, Tianjin Medical University General Hospital, 154 Anshan Road, Heping District, Tianjin 300052, P.R. China

E-mail: zhongyue2457@sina.com

Dr Chunsheng Kang, Laboratory of Neuro-Oncology, Tianjin Neurological Institute, 154 Anshan Road, Heping District, Tianjin 300052, P.R. China

E-mail:kang97061@yahoo.com

*Contributed equally

Key words: glioma, microRNA-451, target gene, calcium binding protein 39 likely through targeting CAB39 directly and inhibiting the $\mathrm{PI} 3 \mathrm{~K} / \mathrm{AKT}$ pathway indirectly.

\section{Introduction}

Glioblastoma multiforme (GBM) is the most common, lethal and aggressive type of primary brain tumor with a median survival of 9-12 months. Despite advances in the basic understanding of cancer biology and therapeutic advances in other neoplasms, the poor prognosis for GBM has not improved in the last four decades and its treatment remains ineffective and essentially palliative $(1,2)$. Though the exact molecular mechanisms for glioma genesis remain unclear, recent studies have reported that there were several microRNA (miRNA or miR) abnormalities in human gliomas, including miR-451 (3). miRNAs are now being used as a new type of molecules of interest to elucidate tumorigenesis and novel therapeutic approaches could be developed by targeting miRNAs that are altered in glioma (4).

miRNAs are a class of small ( 22 nucleotides) non-coding RNAs that function as negative regulators of gene expression at the post-transcriptional level by binding to complimentary sequences in, mainly, the 3'-untranslated regions (3'-UTRs) of specific mRNAs (5-11). These miRNAs play important roles in apoptosis, proliferation, differentiation, development, and metabolism (12-14). In particular, miRNAs show altered expression in tumors in relation to normal tissues and miRNA aberrations may be important in tumor progression (15). hsa-miR-451 is located on chromosome 17q11.2, a region known to be amplified in certain types of cancers, in close proximity to ERBB2 (17q12) (16,17). Studies have shown that miR-451 inhibited cell growth (3), proliferation, and invasion and enhance apoptosis (18). The identification of target genes associated with altered miRNA expression might accurately elucidate the role of miRNAs in cancer biology (15).

In this study, we confirmed the hypo-expression of miR-451 in gliomas using quantitative real-time PCR and fluorescent in situ hybridization. The calcium binding protein 39 gene (CAB39) predicted by bioinformatics analysis as a target gene of the miR-451, was validated by fluorescent reporter assay. We further analyzed the signaling pathway miR-451 might regulate in human glioma and found that miR-451 modulated the expression of multiple downstream molecules such as LKB1, AMPK, 
PI3K and AKT, suggesting that miR-451 may act as a tumorsuppressor factor and regulate the PI3K/AKT pathway through LKB1 and AMPK.

\section{Materials and methods}

Patients and samples. Tissue specimens and clinical information were obtained as part of an approved study by the Institutional Review Board at the Tianjin Medical University, China. Forty-six human glioma tissues were collected with patient consent at the time of operation, grading of tumors was carried out with WHO criteria (World Health Organization, 2007). The tissue samples included: 3 grade I tumors (3 hairy cell astrocytoma); 8 grade II tumors (1 protoplasmic astrocytomas, 6 fibrocytic astrocytomas and 1 mixed oligoastrocytomas); 15 grade III gliomas (all of these tumors were anaplastic astrocytomas); and 15 grade IV glioblastomas (GBMs). Five normal brain tissues were obtained from patients with traumatic brain injury and brain tumors for internal decompression. Immediately after surgery, samples were snap-frozen and stored in liquid nitrogen.

A glioma tissue microarray was purchased from Shaanxi Chaoying Biotechnology (Xi'an, China). Pathologic grades of tumors on the microarray were defined according to the 2007 WHO criteria as follows: 4 grade I tumors (4 Pilocytic astrocytoma), 18 grade II tumors (15 astrocytoma, 2 oligoastrocytoma and 1 oligodendroglioma), 14 grade III tumors (10 anaplastic astrocytoma, 3 anaplastic oligoastrocytoma and 1 anaplastic oligodendroglioma), 39 grade IV tumors (all glioblastomas); 5 were normal brain tissue. Each dot represented a tissue spot from one individual specimen, selected and pathologically confirmed. The array dot diameter was $1.5 \mathrm{~mm}$. All microarrays were stored in the dark at $4^{\circ} \mathrm{C}$.

In situ hybridization. Using sense locked nucleic acid (LNA)modified oligonucleotide probes, in situ hybridization was performed with an in situ hybridization kit (Boster Biological Technology, Ltd., Wuhan, China). The sequences of the LNA/ DNA oligonucleotides contained locked nucleic acids at five consecutive centrally located bases (indicated by the underline) as shown: HSA-miR-451 5'-TTGAG TCATT ACCAT TGCCA AA-3'. The glioma tissue microarrays were deproteinated, and then prehybridized for $2 \mathrm{~h}$ in hybridization liquid in a humidified chamber ( $50 \%$ formamide, 5 x SSC). The probes (miR-451 $10 \mathrm{ng}$ ) were added to the sections on the microarray and incubated overnight at $40^{\circ} \mathrm{C}$ in a water bath. After washing with PBS 3 times, the same volume of anti-digoxigenin-rhodamine and streptavidin-FITC solution was added and incubated for $2 \mathrm{~h}$ at room temperature in the dark. Nuclei were counterstained with a DAPI karyotyping kit (Genmed, USA). After washing with PBS 3 times, sections were sealed and detected under a fluorescence microscope with an OptiGrid system and analyzed by IPP6.1 (Olympus, Tokyo, Japan).

Analysis of hsa-miR-451 candidate target genes. Previous studies in our laboratory have shown a negative correlation between miR-451 and the expressions of AKT1 and c-Myc (18). We further analyzed our data using several databases (Fig. 1). First, we used TargetScan 5.1 (http://www.targetscan. org) to search for candidate miR-451 target genes and found 14 (YTHDF2, ZNF644, CUGBP2, C11orf30, FMNL3, FBXO33,
AKTIP, VAPA, RKHD2, SAMD4B, OSR1, TTN, CAB39, YWHAZ). Next, we used STRING, the functional protein association networks database (http://www.bork.embl-heidelberg. de/STRING/) (19), to explore possible interactions between the 14 candidate miR-451 target genes and AKT1 and c-Myc. To exploit the possible interactions that were identified between the miR-451 target genes and AKT1 and c-Myc we used the KEGG pathway database (http://www.genome.jp/kegg/) (20). For a second prediction of possible hsa-miR-451 (human miR-451) gene targets we used MicroCosm Targets (http://microrna. sanger.ac.uk/) (21) and investigated whether AKT1 and c-Myc were included in the prediction. Finally, to further evaluate the possibility of AKT1 and c-Myc as hsa-miR-451 target genes we used RNAhybrid (http://bibiserv.techfak.uni-bielefeld.de/ rnahybrid) (22).

Cell culture and transfection. The human GBM cell lines LN229, U87 and U251 were purchased from the Institute of Biochemistry and Cell Biology, Chinese Academy of Sciences, Shanghai, China. The human glioblastoma cell line A172 was gifted by Professor Jinhuan Wang (Tianjin First Central Hospital, China). The cells were maintained in Dulbecco's modified Eagle's medium (DMEM, Gibco, USA) supplemented with $12 \%$ fetal bovine serum (Invitrogen, Carlsbad, USA), and incubated at $37^{\circ} \mathrm{C}$ with $5 \% \mathrm{CO}_{2}$. Transfections with hsa-miR-451 mimics were performed in serum-free medium $24 \mathrm{~h}$ after plating, with Lipofectamine 2000 (Invitrogen). The oligonucleotide sequence of the hsa-miR-451 mimics was: 5'-AAACCGUUACCAUUACUGAGUU-3'. A scrambled siRNA sequence (5'-TTCTCCGAACGTGTCACGT-3') was used as the negative control (Gima Biol Engineering Inc., Shanghai, China). Cells were then cultured in complete medium $6 \mathrm{~h}$ later.

Quantative real-time PCR analysis. Total RNA of the GBM cells (U251,LN229, A172, U87), as well as of the 46 snap-frozen human glioma tissues was harvested using TRIzol (Invitrogen) following the manufacturer's protocol. The concentration and purity of RNA were determined using NanoDrop ${ }^{\circledR}$ ND-1000. Total RNA ( $2 \mu \mathrm{g}$ ) was used for cDNA synthesis by reverse transcription using M-MLV Reverse Transcriptase (Promega) according to the manufacturer's protocol. Expressions of mature miR-451 were quantified by miR-qRT PCR using the Hairpin-it $^{\mathrm{TM}}$ miRNA qPCR Quantitation kit (GenePharma Co. Ltd.). All PCR reactions were performed using standard PCR conditions: stage $1: 95^{\circ} \mathrm{C}$ for $3 \mathrm{~min}\left(1\right.$ cycle); stage $2: 95^{\circ} \mathrm{C}$ for $12 \mathrm{sec}$, followed by $62^{\circ} \mathrm{C}$ for $40 \mathrm{sec}$; stage 3: from $62^{\circ} \mathrm{C}$ up to $95^{\circ} \mathrm{C}$, followed by $0.2^{\circ} \mathrm{C}$ for $2 \sec (1$ cycle). U6 was used as the internal control. Data are shown as fold change and analyzed initially using Opticon Monitor Analysis Software V2.02 software (MJ Research, Waltham, MA, USA).

Luciferase activity assay. The pGL3-CAB39-3'UTR-Subcloning and pGL3-CAB39-3'UTR-Mut plasmids were purchased from GenScript (Nanjing, China). The cDNA was cloned into the $X b a \mathrm{I} / X b a \mathrm{I}$ site of the pGL3-control vector downstream of the luciferase gene, to generate pGL3-CAB39 vectors with the following oligonucleotide sequences (Fig. 3C): The gene CAB39 3'UTR-Wild: 3'-TCTAGATGTTAGCTATTCAG CATCAGGCACTCTTATTGATTCATGAGGAACACTGC 
$\bullet$

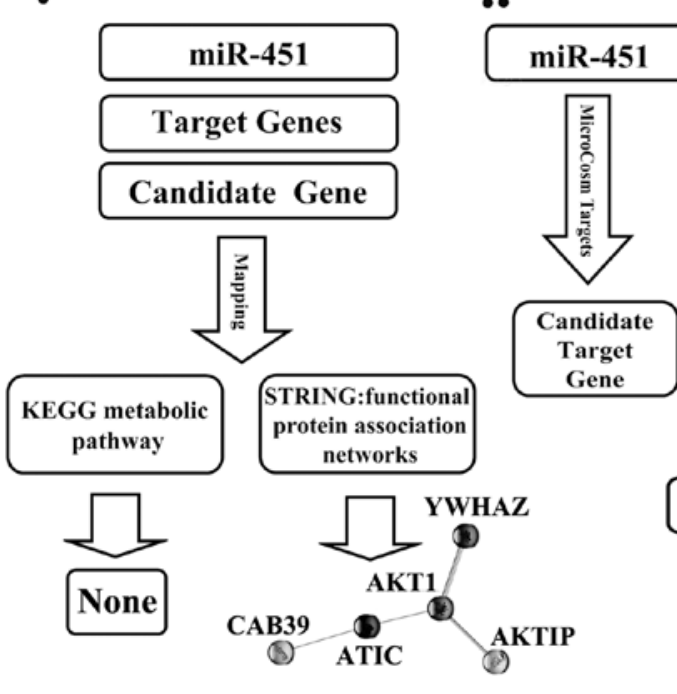

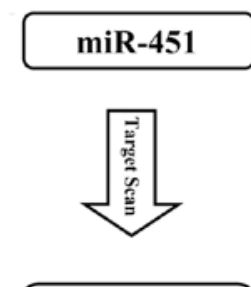

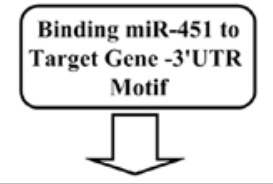

Candidate Target Gene

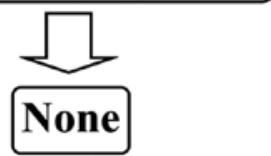

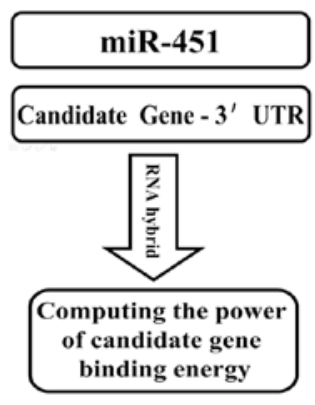

Figure 1. Pipeline for the analysis of the hsa-miR-451 candidate target genes using miRNA target prediction software (TargetScan and MicroCosm Targets), STRING, the functional protein association network database, the KEGG pathway database and RNAhybrid.
TAATCTGCTGTTAAGTGAACGGTTTTTCATTTTACCCT TTTGTTTTTCAGTCCAGGTTGGAGATCGTAGCTGCTG CTGCTTGCACACTCTAGAA-5'; The gene CAB39 3'UTRMut: 3'-TGTTAGCTATTCAGCATCAGGCACTCTTATTGA TTCATGAGGAACATTACTGCTAATCTGCTGTTAAGTGC CATTGGGTTCATTTTACCCTTTTGTTTTTCAGTCCAGG TTGGAGATCGTAGCTGCTGCTGCTTGCACACC-5'.

For the luciferase reporter assay, the U251, LN229, U87 and A172 cells were cultured in 96-well plates (2000 cells per well), transfected with 5 pmol of the hsa-miR-451 mimic oligonucleotide with Lipofectamine 2000. $24 \mathrm{~h}$ after transfection, the cells were transfected again, this time with $0.2 \mu \mathrm{g}$ of either the pGL3-CAB39-3-UTR-Subcloning plasmids or the pGL3-CAB39-3'UTR-Mut plasmids with Lipofectamine 2000. After $48 \mathrm{~h}$ of this transfection, luciferase activity was measured using the Luciferase Assay System (Promega).

Western blot analysis. Cells were harvested $48 \mathrm{~h}$ after transfection, rinsed three times with ice-cold PBS and then extracted with $1 \%$ Nonidet P-40 lysis buffer (20 mM Tris, $\mathrm{pH} 8.0,137 \mathrm{mM} \mathrm{NaCl}$, $1 \%$ Nonidet P-40, $10 \%$ glycerol, $1 \mathrm{mM} \mathrm{CaCl} 2,1 \mathrm{mM} \mathrm{MgCl}_{2}, 1$ $\mathrm{mM}$ phenylmethylsulfonyl fluoride, $1 \mathrm{mM}$ sodium fluoride, 1 $\mathrm{mM}$ sodium orthovanadate, and a protease inhibitor mixture). Homogenates were clarified by centrifugation at $20,000 \times \mathrm{g}$ for $15 \mathrm{~min}$ at $4^{\circ} \mathrm{C}$, and protein concentrations were measured by Nanodrop spectrophotometer (Gene, USA). Protein lysates $(50 \mu \mathrm{g})$ from each sample were subjected to sodium dodecyl sulfate-polyacrylamide gel electrophoresis (SDS-PAGE) on $10 \%$ SDS polyacrylamide gel. The separated proteins were transferred to polyvinylidene difluoride (PVDF) membranes (Millipore, Billerica, MA). The membrane was incubated with primary antibodies against CAB39, LKB1, AMPK, p-AMPK, PI3K (p110 $\alpha$ ) and p-AKT1/2/3 (1:1000 dilution, Santa Cruz, USA), followed by incubation with an HRP-conjugated secondary antibody (1:1000 dilution, Zhongshan Bio Corp, Beijing, China). The specific protein was detected using a SuperSignal protein detection kit (Pierce, USA). After washing with stripping buffer, the membrane was re-probed with antibody against GAPDH (1:1000 dilution, Santa Cruz).

Subcutaneous tumor assay. BALB/c-A 6-week-old nude mice were purchased from the animal center of the Cancer Institute of Chinese Academy of Medical Science and housed individually in ventilated microisolator cages with water and food. All experimental procedures were carried out according to the regulations and internal biosafety and bioethics guidelines of Tianjin Medical University and the Tianjin Municipal Science and Technology Commission. The LN229 subcutaneous tumor xenograft model was established as previously described (23). When the tumors were approximately $5 \mathrm{~mm}$ in length, the mice were randomly divided into 3 groups (10 mice per group): the LN229 control group, the LN229 scramble PBS-treated group, and the LN229 miR-451-treated group. A mixture of $5 \mu 1$ oligonucleotides containing scramble miR-451 mimics and $10 \mu 1$ Lipofectamine was injected into the xenograft tumor model in a multi-site injection manner. The mice in the LN229 control group received $10 \mu \mathrm{l}$ of PBS only. Treatment was conducted every four days, until the end of the experiment. The tumor volume was measured with a caliper every 3 days using the formula, volume $=$ length $\mathrm{x}$ width $/ 2$. At the end of a 21-day observation period, the mice were sacrificed and the tumor tissues were removed for formalin fixation and preparation of paraffin-embedded sections for immunohistochemical analysis.

Immunohistochemistry analysis. The paraffin-embedded tissue sections were examined for CAB39, AMPK, p-AMPK, LKB1, PI3K, p-AKT, and GAPDH expression, while the glioma tissue microarrays were examined for CAB39 expression, and $\mathrm{H} \& \mathrm{E}$ staining. Sections were dewaxed, treated with $3 \% \mathrm{H}_{2} \mathrm{O}_{2}$ for $10 \mathrm{~min}$, and incubated with appropriate primary antibodies (1:100; Santa Cruz Biotechnology) overnight at $4^{\circ} \mathrm{C}$. They were then treated with biotinylated secondary antibody $(1: 100)$ for $1 \mathrm{~h}$ at room temperature, followed by incubation with avidin-biotin complex (ABC)-peroxidase for a further $1 \mathrm{~h}$. After washing 


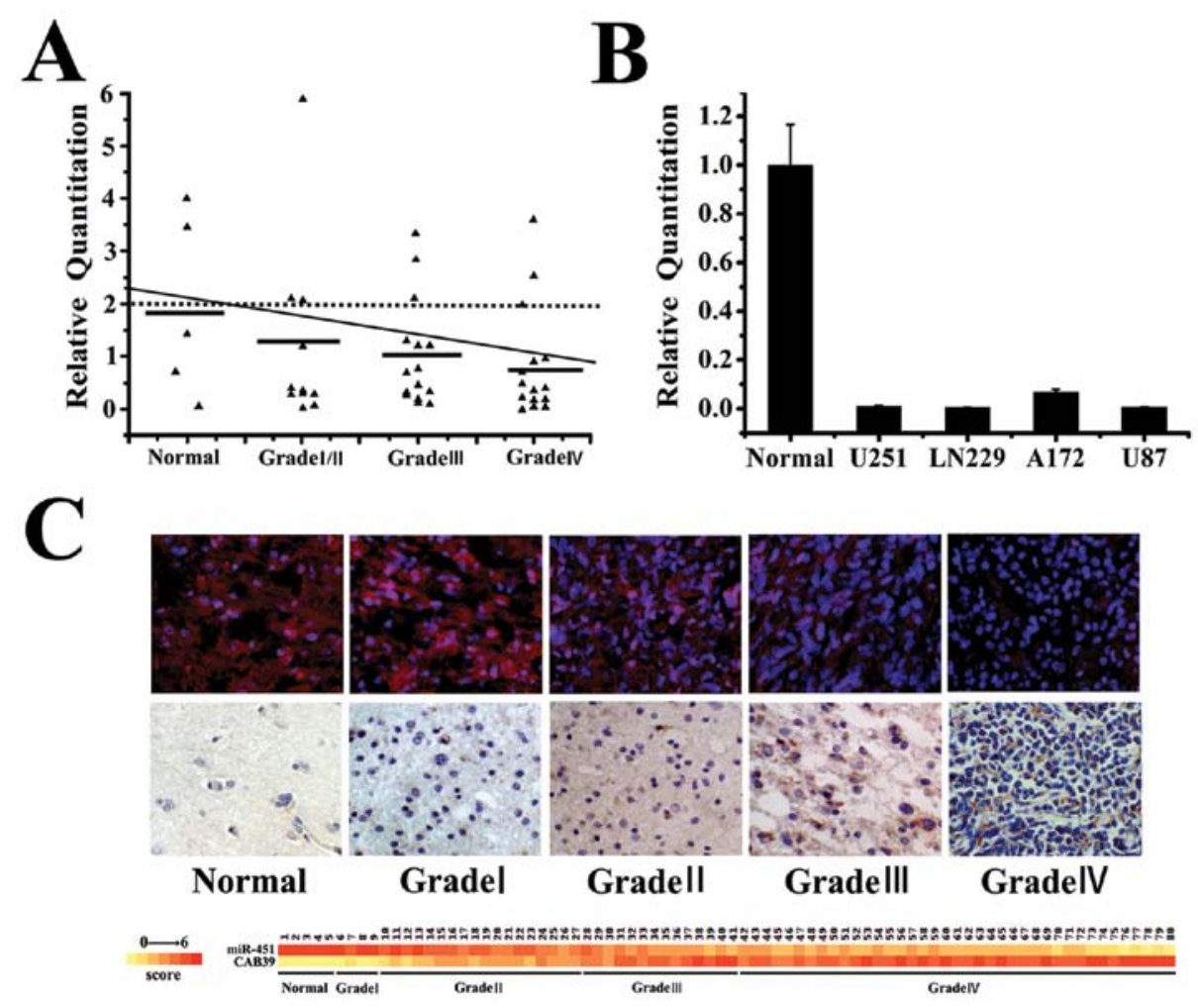

Figure 2. The in vitro and in vivo relationships between miR-451 expression and glioma grades. (A and B) The correlation between miR-451 expression and glioma WHO grades by qRT-PCR. (C) The correlation between miR-451 expression and glioma WHO grades by in situ hybridization. (A and C) Normal brain tissues and gliomas of different WHO grades were compared; (B) normal brain tissues and four different glioblastoma cell lines were compared.

with Tris buffer, the sections were incubated with 3,3'-diaminobenzidine (DAB) for $5 \mathrm{~min}$, rinsed in water, counterstained with hematoxylin, and visualized using a light microscope.

Statistical analysis. SPSS10.0 was used for statistical analysis. One-way analysis of variance (ANOVA) and $\chi^{2}$ test was used to analyze the significance between groups. The LSD method of multiple comparisons with parental and control vector groups was used when the probability for ANOVA was statistically significant. Statistical significance was determined at $\mathrm{p}<0.05$.

\section{Results}

miR-451 expression was negatively correlated with the WHO grades of gliomas. To further study the biological role of miR-451 in human glioma tissues, we examined miR-451 expression in normal brain tissues, glioma tissues and glioma cell lines by quantitative RT-PCR. As shown (Fig. 2A and B), miR-451 expression decreased with the increasing WHO grades of glioma tissues. In situ hybridization analysis revealed that miR-451 was expressed in gliomas and its total positive rate was $94.67 \%$ (71/75). The expression of miR-451 decreased markedly in high grade gliomas (WHO grades III and IV) compared to its expression in low grade gliomas (WHO grades I and II). Indeed, 22/22 low grade gliomas exhibited detectable levels of miR-451, while in 4/53 high grade gliomas miR-451 was at detectable levels $(\mathrm{p}<0.05)$ (Fig. 2C).

$C A B 39$ is a target gene of miR-451 in glioma. Although the reported under-expression of miR-451 in some types of tumors suggested a role in cancer development, the underlying mechanism is still unclear because little is known about the miR-451 target genes. Therefore, the identification of miR-451-regulated targets is a necessary step to understand how miR-451 functions. We used a three-step consequential approach to identify miR-451 target genes. First, target genes were predicted by bioinformatics analysis, then, predicted genes were tested with Western blotting and finally, potential target genes were validated by fluorescent reporter assay (25).

miR-451 target gene(s) identification. Bioinformatics analysis failed to identify AKT1 and/or c-Myc as hsa-miR-451 target genes. However, using the STRING proteins functional association network database, AKT1 was shown to have a direct association with AKTIP and YWHAZ and an indirect association with $\mathrm{CAB} 39$, all three of these genes were predicted to be hsa-miR-451 target genes (Fig. 1). CAB39-AMPK-mTOR-AKT1 was inferred in KEGG pathway database, with CAB39AMPK-mTOR documented while AKT1 not. It is possible that hsa-miR-451 interacts indirectly with AKT1, through its target genes. Both the STRING and KEGG databases indicated that CAB39 was the preferential candidate target gene of hsa-miR451.

miR-451 target gene confirmation. Quantitative real-time PCR showed that miR-451 expression increased in U251, LN229, A172, and U87 cells by 340.14, 849.22, 1680.88 and 2033.85fold respectively after transfection with the miR-451 mimics, compared to its expression in the control and scramble treated cells (Fig. 3A). The transfected cells were used in subsequent 


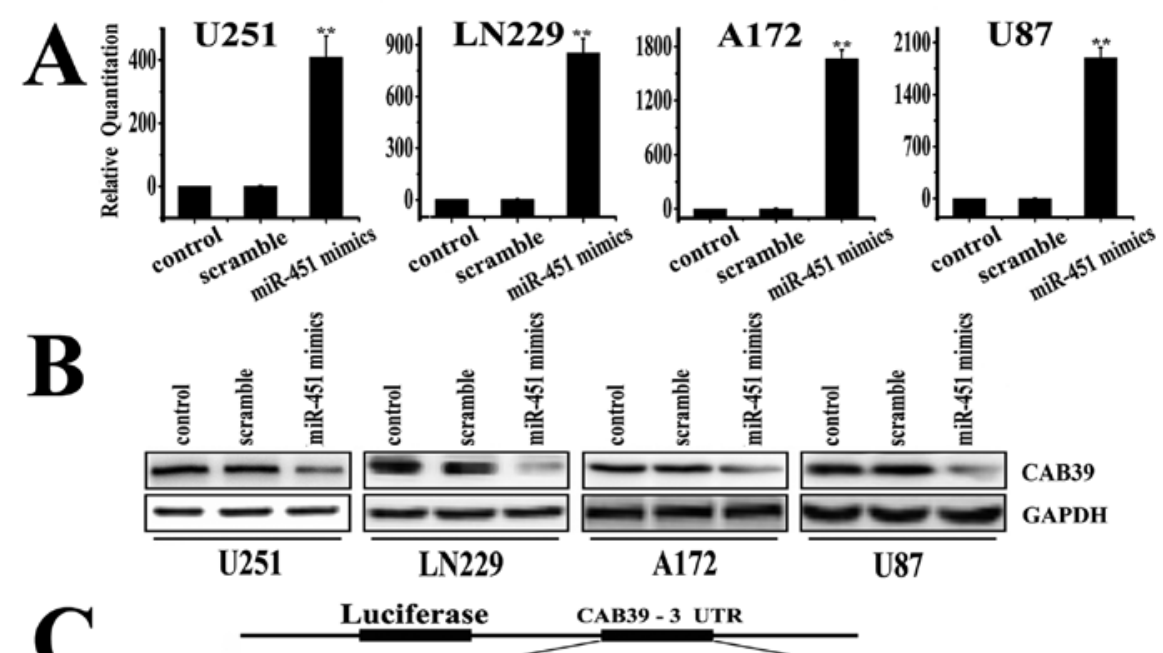

Wild CAB39 UTR 5' AUCUGCUGUUAAGUG -AACGGUUU $3{ }^{\prime}$ Hsa - miR-451 3 ' UUGAGUCAUUACCAUUGCCAAA 5 Mut CAB39 UTR 5' AUCUGCUGUUAAGUG - CCAUUGGG 3' Hsa - miR - 451 3' UUGAGUCAUUACCAUUGCCAAA 5
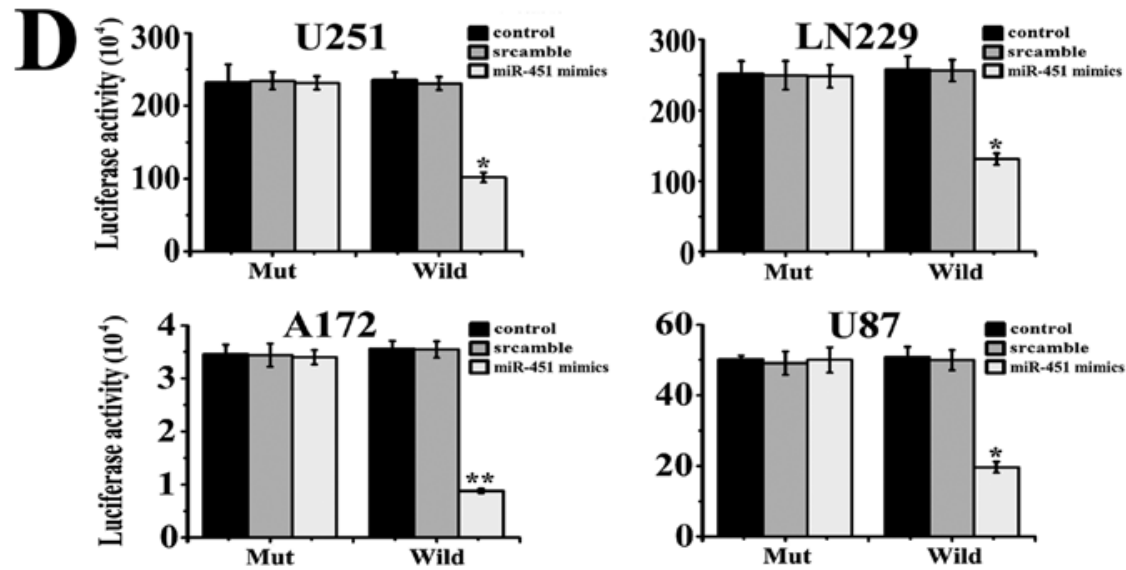

Figure 3. Analysis of CAB39 as a target gene of the miR-451. (A) Relative quantitation of CAB39, using quantitative real-time PCR, in U251, LN229, A172, and U87 cell lines following transfection with the miR-451 mimics ( $\left.{ }^{*}<0.05 ;{ }^{* *} \mathrm{p}<0.01\right)$. (B) Western blot analysis showing CAB39 in the hsa-miR-451-mimics treated cells. (C) Schematic representation of the pGL3-CAB39 3'UTR containing reporter constructs. (D) Luciferase activity in the human glioma cells co-transfected with hsa-miR-451 and the pGL3-CAB39-3'UTR plasmids ( $\mathrm{p}=0.0011)$.

Western blot assays and CAB39 was found to be significantly downregulated in cells treated with the hsa-miR-451 mimic oligonucleotide. Finally, as shown in Fig. 3B, luciferase activity was significantly decreased in cells co-transfected with hsa-miR451 and pGL3-CAB39-3'UTR-wild plasmid cells compared to its activity in the scramble, negative control and pGL3-CAB393'UTR-Mut plasmid-treated cells ( $\mathrm{p}=0.0011$, Fig. 3D). Together, these data demonstrated that CAB39 is a target gene of the miR-451 in glioma.

Previously we have shown that miR-451 expression was negatively correlated with glioma WHO grades in quantitative real-time PCR and in situ hybridization. Here, we found a similar phenomenon between CAB39 expression and glioma WHO grades (Fig. 2C), using immunohistochemistry. CAB39 was expressed in gliomas and its total positive rate was $96 \%$ (72/75). The levels of CAB39 increased markedly in high grade gliomas (WHO grades III and IV) in comparison to low grade gliomas (WHO grades I and II). Indeed, 53/53 high grade gliomas exhibited detectable levels of CAB39, while 3/22 low grade gliomas the protein was at undetectable levels $(\mathrm{p}<0.05)$.
miR-451 regulated PI3K/ATK pathway factors in human glioma in vitro. Previous data from our laboratory showed that miR-451 had a significant impact on cell proliferation, invasion and apoptosis in human glioblastoma cell lines, possibly by regulating AKT expression (18). AKT1 plays a critical role in controlling a diversity of cellular functions, such as protein synthesis, cell cycle, cell survival and apoptosis $(26,27)$. We, therefore, investigated AKT-related pathways. As shown in Fig. 4B, obvious activation of phosphorylated-AKT was observed in U251, LN229, A172 and U87 cells after transfection with the miR-451 mimics. Consistently, over-expression of miR-451 led to a marked downregulation of LKB1, AMPK, p-AMPK, and PI3K, all of which are involved in the pathway upstream of AKT (Fig. 4A). These data suggest that the tumor suppressor activity of miR-451 in glioblastoma cells likely acts through its regulation of the PI3K/ AKT pathway.

miR-451 inhibited the growth of LN229 glioblastoma cells in vivo. To further understand the anti-tumor effect of miR-451 and its role in the signaling pathway in vivo, we employed an 

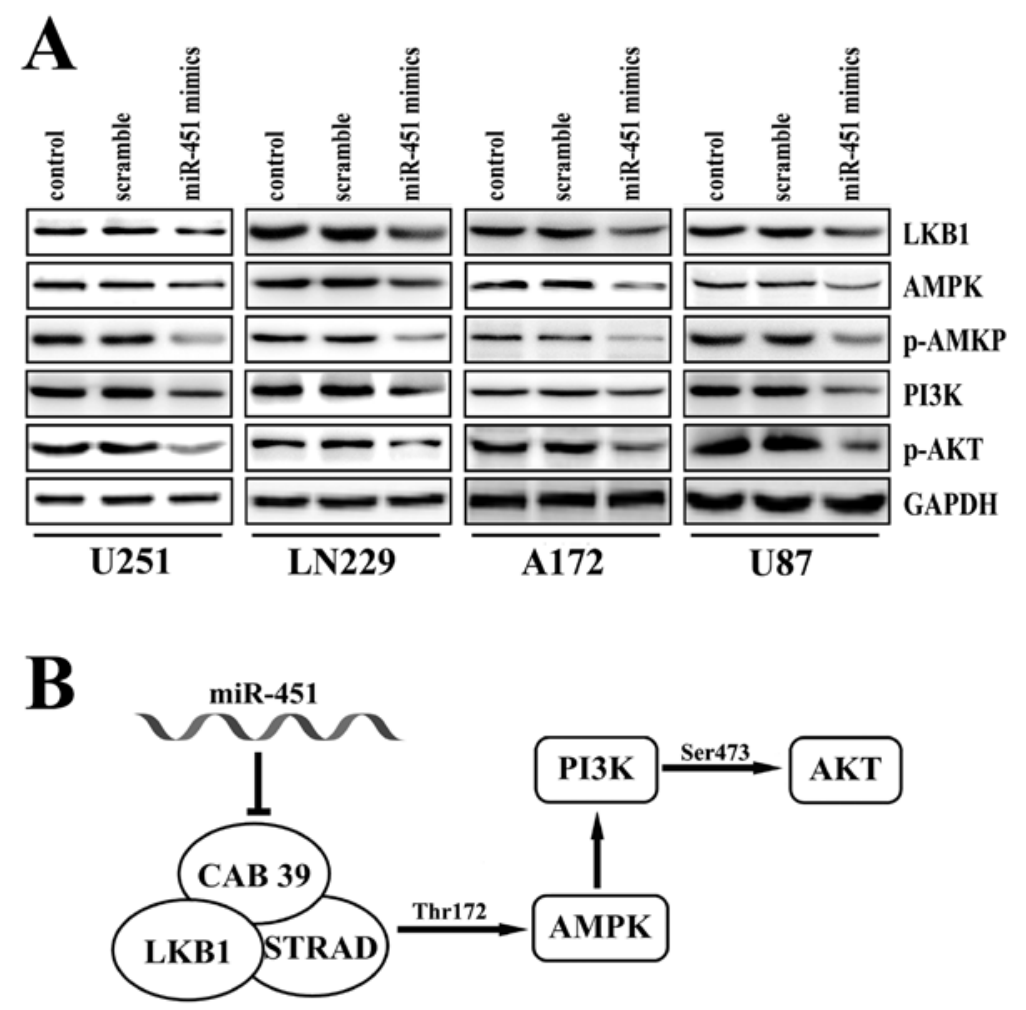

Figure 4. Impact of the miR-451 mimic oligonucleotide on some of the components of the PI3K/AKT signaling pathway. (A) U251, LN229, A172 and U87 cells were treated with the miR-451 mimics and LKB1, AMPK, p-AMPK, PI3K, p-AKT and GAPDH expressions were determined by Western blotting 48 h after treatment. (B) Schematic diagram of how miR-451 downregulation could lead to activation of the PI3K/AKT pathway.

LN229 xenograft glioma mouse model. The mean volume of the tumors used in this study prior to treatment was $56 \pm 20.35 \mathrm{~mm}^{3}$ (Fig. 5B). During the first 3 days of observation following intratumoral administration of miR-451, tumors in both the control and treated groups grew slowly with no marked differences in tumor size between them. Tumors in the group treated with miR-451 maintained a slow growth rate throughout the experiment while tumors in the control group began to grow faster. On day 12, tumors of the mice in the miR-451 treated group started to show statistically significant differences in size compared to tumors of the mice in the control group $(\mathrm{p}<0.05)$. At the termination of the study, the difference in tumor mass between the miR-451 treated group and the control group was marked $(\mathrm{p}<0.01)$. No difference in tumor volume was observed between the control and PBS-treated groups (Fig. 5A). To determine whether intratumoral miR-451 administration affected the expression of factors in the PI3K/AKT signaling pathway, the expressions of CAB39, LKB1, AMPK, p-AMPK, PI3K and $\mathrm{p}-\mathrm{AKT}$ were tested and were found to show significant downregulation in an immunohistopathological examination (Fig. 5C).

\section{Discussion}

miRNA expression profiling studies revealed that a number of miRNAs were dysregulated in human glioblastoma. miRNA451 was one of them. A tumor suppressive role of miR-451 was shown in gliomas in vitro (18), but whether or not miR-451 actually participated in gliomagenesis still needs further inves- tigation. Expression alteration can be a useful index of such activity. We used a high throughput experiment, microarray in situ hybridization examination, and further validated the result using the more quantitative and more sensitive quantitative real-time PCR test on clinical samples. miR-451 was downregulated in glioma tissues and a significant negative correlation was revealed between miR-451 expression and glioma WHO grades.

Fluorescent reporter assay is generally accepted as a gold standard to determine miRNA targets. Using this assay, we demonstrated that CAB39 was a target gene of miR-451. CAB39 (MO25) is an armadillo repeat scaffolding-like protein with two isoforms, CAB39 $\alpha$ and CAB39 $\beta(28,29)$. Structural studies have revealed that the CAB39 $\alpha$ forms an extended $\alpha$-helical repeat rod-like structure, distantly related to the armadillo repeat domain (30). CAB39 is a component of the trimeric LKB1-STRAD-MO25 complex $(29,31)$ and its role is to stabilize the binding of STRAD to LKB1 and re-localize LKB1 from the nucleus to the cytoplasm $(29,32)$. LKB1, a member of the serine/threonine kinase family, regulates cell polarity and functions as a tumor suppressor. Mutations in this widely expressed protein kinase in humans result in a disorder termed Peutz-Jeghers syndrome (PJS), which predisposes the sufferer to a wide spectrum of benign and malignant tumors $(33,34)$. LKB1 is activated through its interaction with STRAD (37) and MO25 (29). AMP-activated protein kinase (AMPK) is a sensor of cellular energy charge which regulates physiological processes that consume or regenerate ATP to restore the energy charge in the cells (35). Studies in mammalian cells demon- 

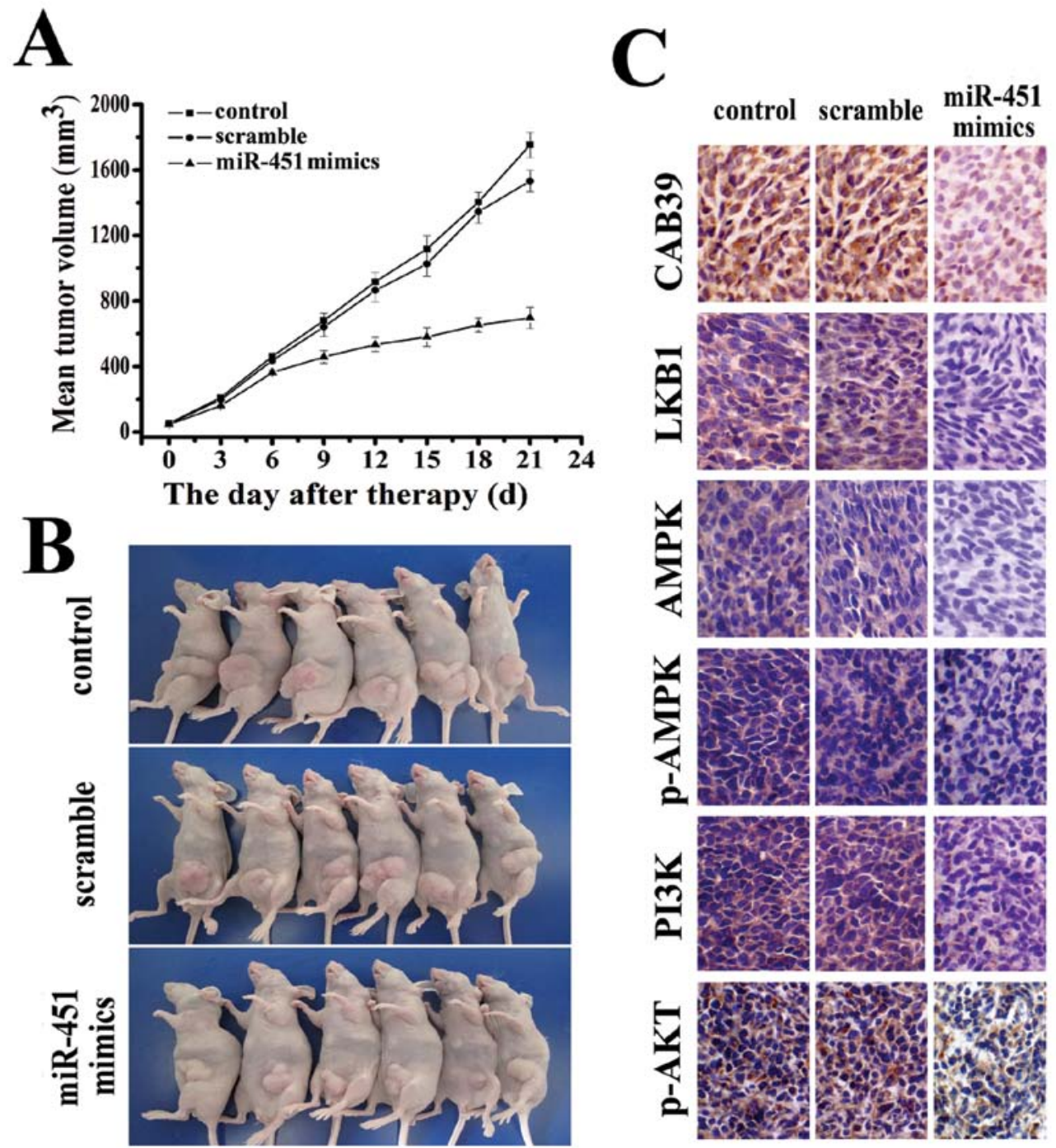

Figure 5. Effects of the upregulation of miR-451 on glioma growth in vivo. (A) In vivo comparison of mean tumor volume after treatment with the miR-451 mimics. (B) Representative animals from the 3 groups at the end of the study. (C) Immunohistochemistry analyses of xenograft tumors after treatment with the miR-451 mimics, comparing the expression levels of CAB39, LKB1, AMPK, p-AMPK, PI3K and p-AKT.

strated that LKB1 complexed to STRAD and MO25 activated AMPK by phosphorylating Thr172, and that the STRAD and MO25 subunits enhanced phosphorylation of AMPK by over 100 -fold $(36,37)$. The phosphatidylinositol-3' kinase (PI3K) family plays complex and extensive roles in many aspects of cell biology and metabolism (38). Signaling through PI3Ks is central to cell survival, growth and proliferation which, as a consequence, is frequently activated in many human cancers, including glioblastoma (39). Data from Luo et al provided new evidence that AMPK activated Akt by regulating PI3K (40).

To verify the significance of LKB1/AMPK, PI3K/AKT signaling in glioma, we performed Western blotting in human glioma cell lines and immunohistochemistry in a tumor xenograft mouse model. Consistently, over-expression of miR-451 led to a marked downregulation of factors upstream of AKT. This evidence, both in vitro and in vivo, implied that miR-451 can suppress cell proliferation in human glioma through the LKB1/AMPK and PI3K/AKT pathway. However, more evidence still needs to be found. Increasing the expression of miR-451 might be a useful therapeutic strategy for treating glioma in the future.

\section{Acknowledgements}

This study was supported by the China National Natural Scientific Fund (Grant no. 81172406), the Tianjin Science and Technology Committee (Grant no. 09JCZDJC20500) and the Technology Fund of the Tianjin Public Health Bureau (Grant no. 09KZ112).

\section{References}

1. Furnari FB, Fenton T, Bachoo RM, et al: Malignant astrocytic glioma: genetics, biology, and paths to treatment. Genes Dev 21: 2683-2710, 2007.

2. Van Meir EG, Hadjipanayis CG, Norden AD, Shu HK, Wen PY and Olson JJ: Exciting new advances in neuro-oncology: the avenue to a cure for malignant glioma. CA Cancer J Clin 60: 166-193, 2010.

3. Gal H,Pandi G, Kanner AA, et al: MIR-451 and imatinib mesylate inhibit tumor growth of glioblastoma stem cells. Biochem Biophys Res Commun 376: 86-90, 2008.

4. Zhang CZ, Zhang JX, Zhang AL, et al: MiR-221 and miR-222 target PUMA to induce cell survival in glioblastoma. Mol Cancer 9: 229, 2010. 
5. Liu T, Tang H, Lang Y, Liu M and Li X: MicroRNA-27a functions as an oncogene in gastric adenocarcinoma by targeting prohibitin. Cancer Lett 273: 233-242, 2009.

6. Moriyama T, Ohuchida K, Mizumoto K, et al: MicroRNA-21 modulates biological functions of pancreatic cancer cells including their proliferation, invasion, and chemoresistance. Mol Cancer Ther 8: 1067-1074, 2009.

7. Selbach M, Schwanhausser B, Thierfelder N, Fang Z, Khanin R and Rajewsky N: Widespread changes in protein synthesis induced by microRNAs. Nature 455: 58-63, 2008.

8. Tay Y,Zhang J, Thomson AM, Lim B and Rigoutsos I: MicroRNAs to Nanog, Oct 4 and Sox 2 coding regions modulate embryonic stem cell differentiation. Nature 455: 1124-1128, 2008.

9. Chi SW,Zang JB, Mele A and Darnell RB: Argonaute HITS-CLIP decodesmicroRNA-mRNAinteractionmaps. Nature 460:479-486, 2009.

10. He L and Hannon GJ: MicroRNAs: small RNAs with a big role in gene regulation. Nat Rev Genet 5: 522-531, 2004.

11. Griffiths-Jones S, Grocock RJ, van Dongen S, Bateman A and Enright AJ: miRBase: microRNA sequences, targets and gene nomenclature. Nucleic Acids Res 34: D140-D144, 2006.

12. Ambros V: The functions of animal microRNAs. Nature 431: 350-355, 2004

13. Bartel DP: MicroRNAs: genomics, biogenesis, mechanism, and function. Cell 116: 281-297, 2004.

14. Hwang HW and Mendell JT: MicroRNAs in cell proliferation, cell death, and tumorigenesis. Br J Cancer 94: 776-780, 2006.

15. Bandres E, Agirre X, Ramirez N, Zarate R and Garcia-Foncillas J: MicroRNAs as cancer players: potential clinical and biological effects. DNA Cell Biol 26: 273-282, 2007.

16. Mahlamaki EH, Barlund M, Tanner M, et al: Frequent amplification of $8 \mathrm{q} 24,11 \mathrm{q}, 17 \mathrm{q}$, and $20 \mathrm{q}$-specific genes in pancreatic cancer. Genes Chromosomes Cancer 35: 353-358, 2002.

17. Varis A, Wolf M, Monni O, et al: Targets of gene amplification and overexpression at 17q in gastric cancer. Cancer Res 62: 2625-2629, 2002.

18. Nan Y, Han L, Zhang A, et al: MiRNA-451 plays a role as tumor suppressor in human glioma cells. Brain Res 1359: 14-21, 2010.

19. von Mering C, Huynen M, Jaeggi D, Schmidt S, Bork P and Snel B: STRING: a database of predicted functional associations between proteins. Nucleic Acids Res 31: 258-261, 2003.

20. Kanehisa $M$ and Goto S: KEGG: kyoto encyclopedia of genes and genomes. Nucleic Acids Res 28: 27-30, 2000

21. Griffiths-Jones S, Saini HK, van Dongen S and Enright AJ miRBase: tools for microRNA genomics. Nucleic Acids Res 36 D154-D158, 2008

22. Kruger J and Rehmsmeier M: RNAhybrid: microRNA target prediction easy, fast and flexible. Nucleic Acids Res 34: W451-W454, 2006.

23. Kang CS, Zhang ZY, Jia ZF, et al: Suppression of EGFR expression by antisense or small interference RNA inhibits U251 glioma cell growth in vitro and in vivo. Cancer Gene Ther 13 $530-538,2006$
24. Zhang J, Han L, Zhang A, et al: AKT2 expression is associated with glioma malignant progression and required for cell survival and invasion. Oncol Rep 24: 65-72, 2010.

25. Wan HY, Guo LM, Liu T, Liu M, Li X and Tang H: Regulation of the transcription factor NF-kappaB1 by microRNA-9 in human gastric adenocarcinoma. Mol Cancer 9: 16, 2010.

26. Brazil DP, Park J and Hemmings BA: PKB binding proteins. Getting in on the Akt. Cell 111: 293-303, 2002.

27. Dufour G,Demers MJ, Gagne D, et al: Human intestinal epithelial cell survival and anoikis. Differentiation state-distinct regulation and roles of protein kinase B/Akt isoforms. J Biol Chem 279: 44113-44122, 2004.

28. Alessi DR, Sakamoto K and Bayascas JR: LKB1-dependent signaling pathways. Annu Rev Biochem 75: 137-163, 2006.

29. Boudeau J, Baas AF, Deak M, et al: MO25alpha/beta interact with STRADalpha/beta enhancing their ability to bind, activate and localize LKB1 in the cytoplasm. EMBO J 22: 5102-5114, 2003.

30. Milburn CC, Boudeau J, Deak M, Alessi DR and van Aalten DM: Crystal structure of MO25 alpha in complex with the $\mathrm{C}$ terminus of the pseudo kinase STE20-related adaptor. Nat Struct Mol Biol 11: 193-200, 2004

31. Brajenovic M, Joberty G, Kuster B, Bouwmeester T and Drewes G: Comprehensive proteomic analysis of human Par protein complexes reveals an interconnected protein network. J Biol Chem 279: 12804-12811, 2004.

32. Baas AF, Boudeau J, Sapkota GP, et al: Activation of the tumour suppressor kinase LKB1 by the STE20-like pseudokinase STRAD. EMBO J 22: 3062-3072, 2003.

33. Hemminki A, Markie D, Tomlinson I, et al: A serine/threonine kinase gene defective in Peutz-Jeghers syndrome. Nature 391: 184-187, 1998.

34. Jenne DE, Reimann H, Nezu J, et al: Peutz-Jeghers syndrome is caused by mutations in a novel serine threonine kinase. Nat Genet 18: 38-43, 1998

35. Hardie DG, Scott JW, Pan DA and Hudson ER: Management of cellular energy by the AMP-activated protein kinase system. FEBS Lett 546: 113-120, 2003

36. Hawley SA, Boudeau J, Reid JL, et al: Complexes between the LKB1 tumor suppressor, STRAD alpha/beta and MO25 alpha/beta are upstream kinases in the AMP-activated protein kinase cascade. J Biol 2: 28, 2003.

37. Woods A, Johnstone SR, Dickerson K, et al: LKB1 is the upstream kinase in the AMP-activated protein kinase cascade. Curr Biol 13: 2004-2008, 2003.

38. Vanhaesebroeck B, Leevers SJ, Ahmadi K, et al: Synthesis and function of 3-phosphorylated inositol lipids. Annu Rev Biochem 70: 535-602, 2001 .

39. Cheng CK, Fan QW and Weiss WA: PI3K signaling in gliomaanimal models and therapeutic challenges. Brain Pathol 19: 112-120, 2009.

40. Tao R, Gong J, Luo X, et al: AMPK exerts dual regulatory effects on the PI3K pathway. J Mol Signal 5: 1, 2010. 